\section{Psychosocial interventions following self-harm}

\author{
Systematic review of their efficacy in preventing suicide
}

MIKE J. CRAWFORD, OLIVIA THOMAS, NUSR AT KHAN and ELENA KULINSKAYA

\begin{abstract}
Background With almost a million people dying by suicide worldwide each year, reducing the rate of suicidal behaviour is a priority in many countries.
\end{abstract}

Aims To examine whether additional psychosocial interventions following an episode of self-harm reduce the likelihood of subsequent suicide.

Method We conducted a systematic review and meta-analysis of data from randomised controlled trials of interventions for people following selfharm. Likelihood of suicide was compared by calculating the pooled root difference in suicide rate with $95 \%$ confidence intervals.

Results We obtained suicide data from 18 studies with a total population of 3918 . Eighteen suicides occurred among people offered active treatment and 19 among those offered standard care (pooled root difference in suicide rate $0.0,95 \% \mathrm{Cl}-0.03$ to 0.03 ). The overall rate of suicide among people participating in trials was similar to that reported in observational studies of people who self-harm.

Conclusions Results of this metaanalysis do not provide evidence that additional psychosocial interventions following self-harm have a marked effect on the likelihood of subsequent suicide.

Declaration of interest None.
It is estimated that almost one million people die by suicide worldwide each year (World Health Organization, 2005). Reducing the rate of suicidal behaviour is a national service priority in Britain and in many other countries (Department of Health, 2002; World Health Organization, 2002). Efforts to reduce the incidence of suicide have been classified into 'high-risk' approaches, which seek to identify and treat those at greatest risk of suicide, and population-based strategies, which aim to reduce or control environmental factors associated with higher levels of suicidal behaviour (Gunnell \& Frankel, 1994). There is a consensus that efforts to prevent suicide should incorporate a broad variety of high-risk and population-based strategies.

One of the most important high-risk groups consists of people who present to services following an episode of non-fatal self-harm. As many as $1.8 \%$ of people who harm themselves die by suicide in the year following the incident (Owens et al, 2002 ) and as many as $8.5 \%$ die by suicide over a 22-year period (Jenkins et al, 2002). It has been argued that enhanced treatment of those who self-harm could help reduce the overall rate of suicide (Gunnell \& Frankel, 1994; Mann et al, 2005). In a review paper examining the impact of different strategies aimed at preventing suicide, Lewis and colleagues estimated that additional interventions following self-harm might reduce the rate of subsequent suicide by $25 \%$ (Lewis et al, 1997).

The view that psychosocial treatment following self-harm could affect the subsequent rate of suicide was supported by findings from a randomised trial of manual-assisted cognitive therapy for people who self-harm. In the year following randomisation, $5(2.5 \%)$ of 203 patients assigned to standard care and $1(0.5 \%)$ of 199 patients assigned to cognitivebehavioural therapy had died by suicide
(Tyrer et al, 2003). This difference did not reach statistical significance; indeed, the relative rarity of suicide following selfharm means that even large trials of people who self-harm lack sufficient power to explore effects on suicide (Gunnell \& Frankel, 1994).

Although the impact of interventions on the rate of repetition of self-harm has been explored (Van der Sande et al, 1997a; Hawton et al, 2000), the effect of such interventions on the likelihood of subsequent suicide has not been examined. We therefore conducted a systematic review and meta-analysis of randomised controlled trials of psychosocial interventions following self-harm, to estimate their impact on the rate of subsequent suicide.

\section{METHOD}

\section{Search strategy}

We started by identifying previously published meta-analyses and systematic reviews of interventions following self-harm and retrieved the primary studies included in these reviews for further analysis. We then searched the following electronic databases: EMBASE (1969 to February 2005); PsycINFO (1967 to February 2005) and Medline (1966 to February 2005). We used a broad range of search terms based on those developed by Hawton et al (2000), and searched the reference lists of all the relevant papers.

\section{Inclusion criteria}

Studies were eligible for inclusion in the review if they were randomised controlled trials; involved patients who had harmed themselves in the period prior to entry into the trial; and compared additional or enhanced intervention with a form of control or standard care. Where papers met these criteria but did not provide mortality data, we attempted to obtain these data from the authors.

\section{Selection of studies and data extraction}

Two raters independently assessed all papers for possible inclusion in the review. If there was disagreement about whether a study should be included this was discussed in detail with a third reviewer and a decision made on whether or not to include it. Information on the study population and interventions were recorded, and data on 


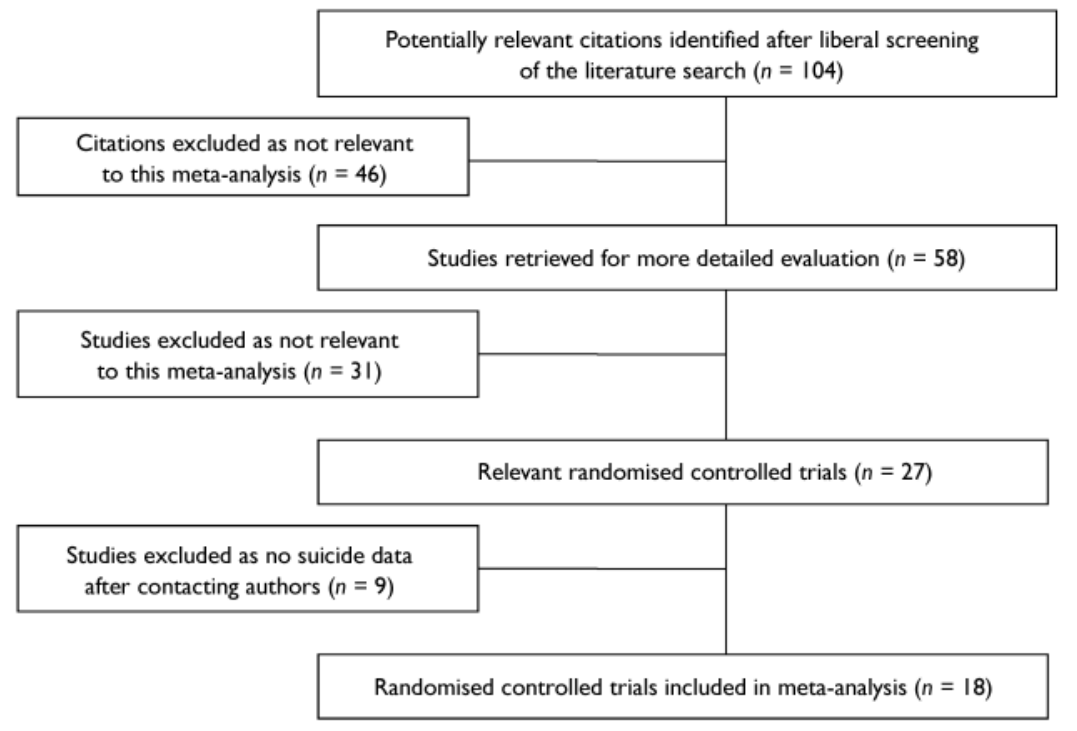

Fig. I Results of literature search and selection of randomised controlled trials.

study size, follow-up rate and numbers of suicides and other deaths in each arm of the trial were extracted by two raters and double-entered onto a database.

\section{Data analysis}

As we were interested in exploring whether additional psychosocial treatment interventions had an impact on the likelihood of suicide, we took an a priori decision to combine data from all psychosocial interventions. Meta-analysis was based on suicide rates; this allowed us to take account of variation in the length of followup of studies (from 3 months to 36 months). Also, if we had used an odds ratio to calculate the overall effect size we would not have been able to incorporate studies in which no suicide was reported. Instead, we calculated the rate of suicide in each arm of the study $\left(\mu_{1}\right.$ and $\left.\mu_{2}\right)$ to model the numbers of deaths $X$ and $Y$ in treatment and control arms. With numbers of participants in the treatment and control arms $m$ and $n$ respectively, the proportion of participants in the control arm of the study who died by suicide $q$ was calculated using the formula $q=n /(m+n)$, and the proportion who died by suicide in the treatment arm by $1-q=m /(m+n)$.

To test for a difference in death rates within each study we can compare a value of a test statistic $T=2\left[(X+3 / 8)^{1 / 2} q^{1 / 2}-\right.$ $\left.(Y+3 / 8)^{1 / 2}(1-q)^{1 / 2}\right]$ to critical values of a standard normal distribution (Huffman, 1984). This statistic is especially useful for our data with low death rates because it uses a variance stabilising transformation. An effect size associated with this statistic is $\theta=\mu_{1}{ }^{1 / 2}-\mu_{2}{ }^{1 / 2}$. Its estimate from a study with a follow-up time $t$ is $\hat{\theta}=[(X+3 / 8) /$ $m t]^{1 / 2}-[(Y+3 / 8) / n t]^{1 / 2}$. The standard deviation of this estimate is $s=2^{-1}[(m+n) /$ $m n t]^{1 / 2}$.

The values of $\theta$ for each study were entered into RevMan 4.2.7 for Windows (Cochrane Collaboration, Oxford, UK; see http://www.cc-ims.net/RevMan) as 'generic inverse variance data' and can be routinely analysed. A fixed-effects model was used. Heterogeneity between studies was examined using the $\chi^{2}$ test. We then repeated the analysis using death from any cause as the outcome. Finally, funnel plot asymmetry was examined in order to assess the possibility of publication bias (Egger $e t$ al, 1997).

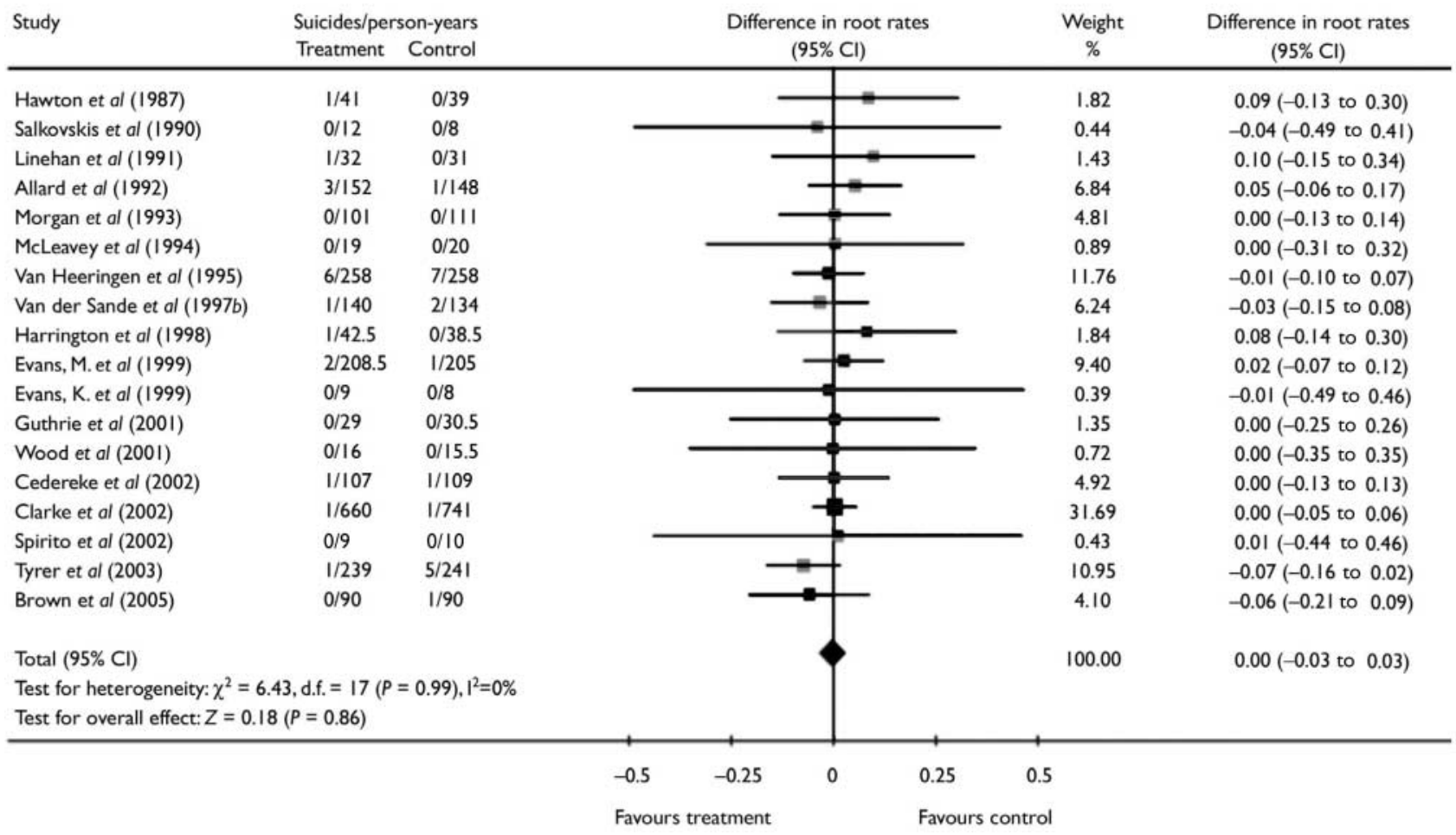

Fig. 2 Meta-analysis of effect of psychosocial interventions on completed suicide. 


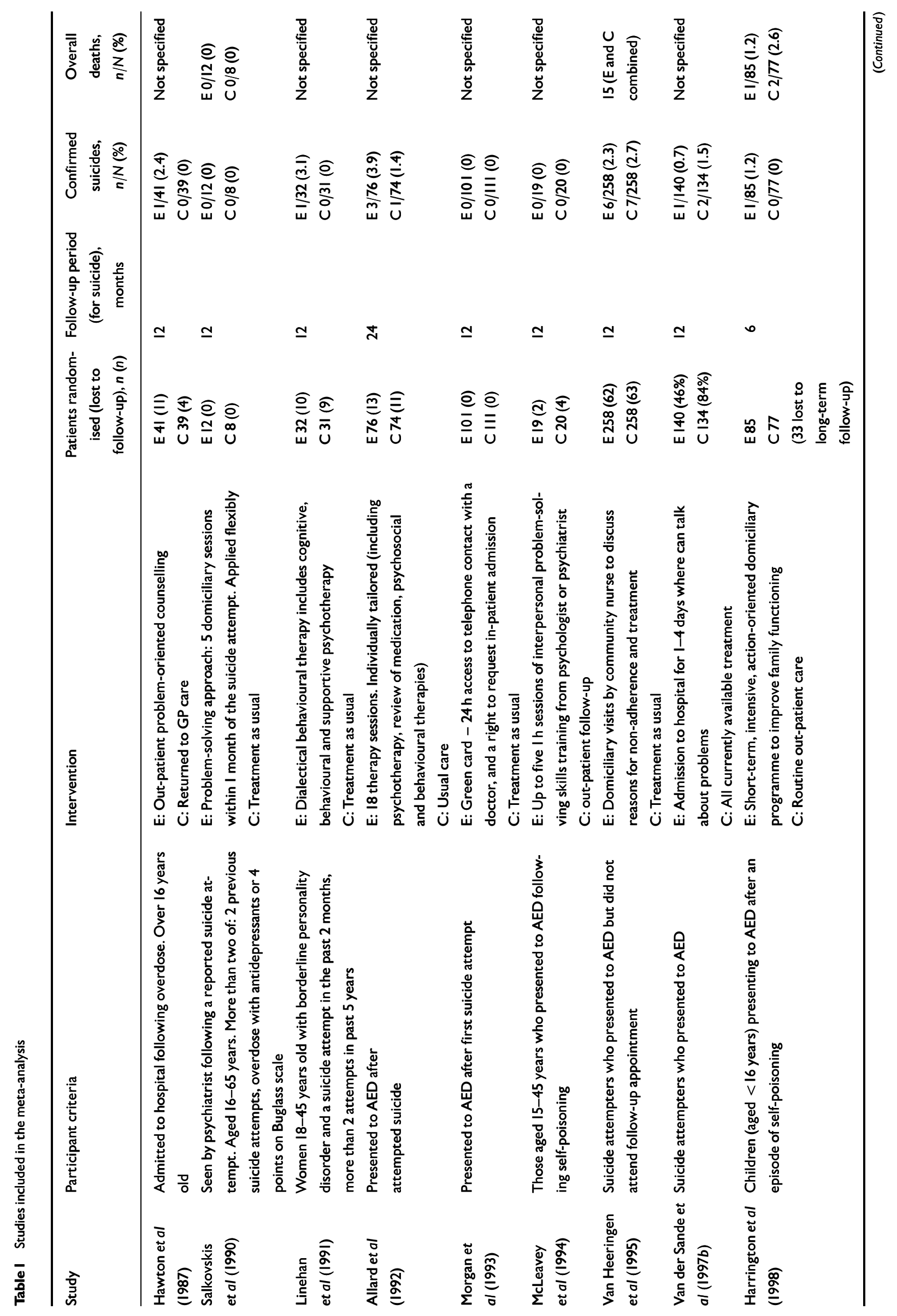




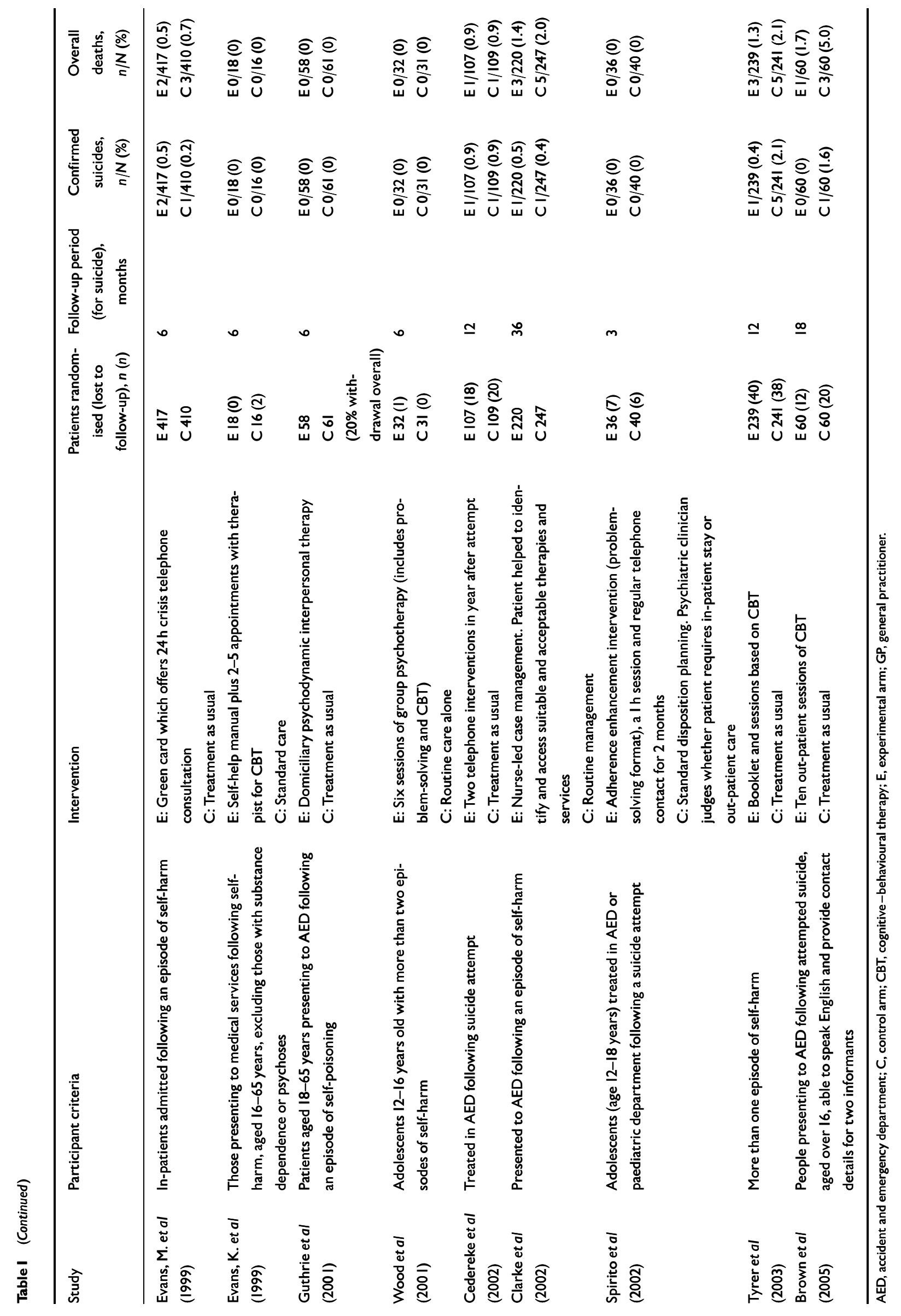




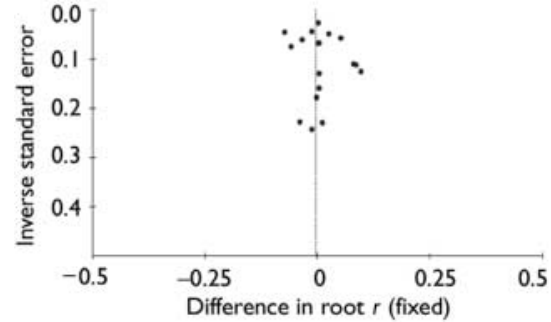

Fig. 3 Funnel plot of studies reporting suicides among those offered experimental and control treatment following episodes of self-harm.

\section{RESULTS}

We initially identified 104 studies for inclusion in the review. Of these, 27 met our inclusion criteria and 12 provided data on suicide deaths. Contact with the studies' authors generated additional data on deaths for six trials and we were therefore able to include 18 trials with a combined population of 3918 participants in the meta-analysis. Figure 1 presents the search process in the form of a QUOROM flow chart (Moher et al, 2000) and Table 1 summarises details of the studies included in the meta-analysis.

\section{Types of interventions}

Most of the interventions involved a limited number (between 2 and 50) of sessions of individual psychotherapy such as cognitive-behavioural therapy, interpersonal psychotherapy and dialectical behaviour therapy. In other studies changes were made to the organisation of services to enhance uptake of psychosocial treatment or facilitate contact with services at times of crisis.

\section{Impact on suicide}

Data from the 18 studies appeared to be homogeneous. Since the value of the test statistic for heterogeneity is less than its degrees of freedom, there was no need to consider the random-effects model. Root differences in rates of suicide in the 18 trials of psychosocial intervention are presented in Fig. 2. Overall there was no evidence of any difference in death rates.

There were 37 suicides among the 3918 participants in the trials of psychosocial interventions, 18 per 2165 person-years among those who received enhanced treatment and 19 per 2237.5 person-years among those who received control treatment. Randomised trials of manual-assisted cognitive therapy (Tyrer et al, 2003), outpatient cognitive-behavioural therapy (Brown et al, 2005), and intensive outpatient care (Van der Sande et al, 1997b) showed a trend towards lower levels of suicide. The remaining studies all showed no difference, or slightly higher levels among those in the active arm of the trial. Funnel plots of effect size estimates against inverse standard error (an indication of study size) were symmetrical, suggesting that publication bias was unlikely to have affected study findings (Fig. 3).

\section{Impact on mortality}

Complete mortality data were available in only 12 studies. Difference in root rates for suicide in the 18 trials of psychosocial intervention are presented in Fig. 4. There was a total of 29 deaths among people in these trials: 18 per 1422 person-years among those receiving control treatment and 11 per 1527.5 person-years among those receiving additional psychosocial interventions.

\section{DISCUSSION}

The results of this meta-analysis provide little evidence to support the view that enhanced treatment following an episode of self-harm substantially reduces the likelihood of subsequent suicide. However, limited power means that our pooled estimate of differences in levels of suicide is imprecise and it is possible that intention is associated with a clinically significant difference in the rate of subsequent suicide.

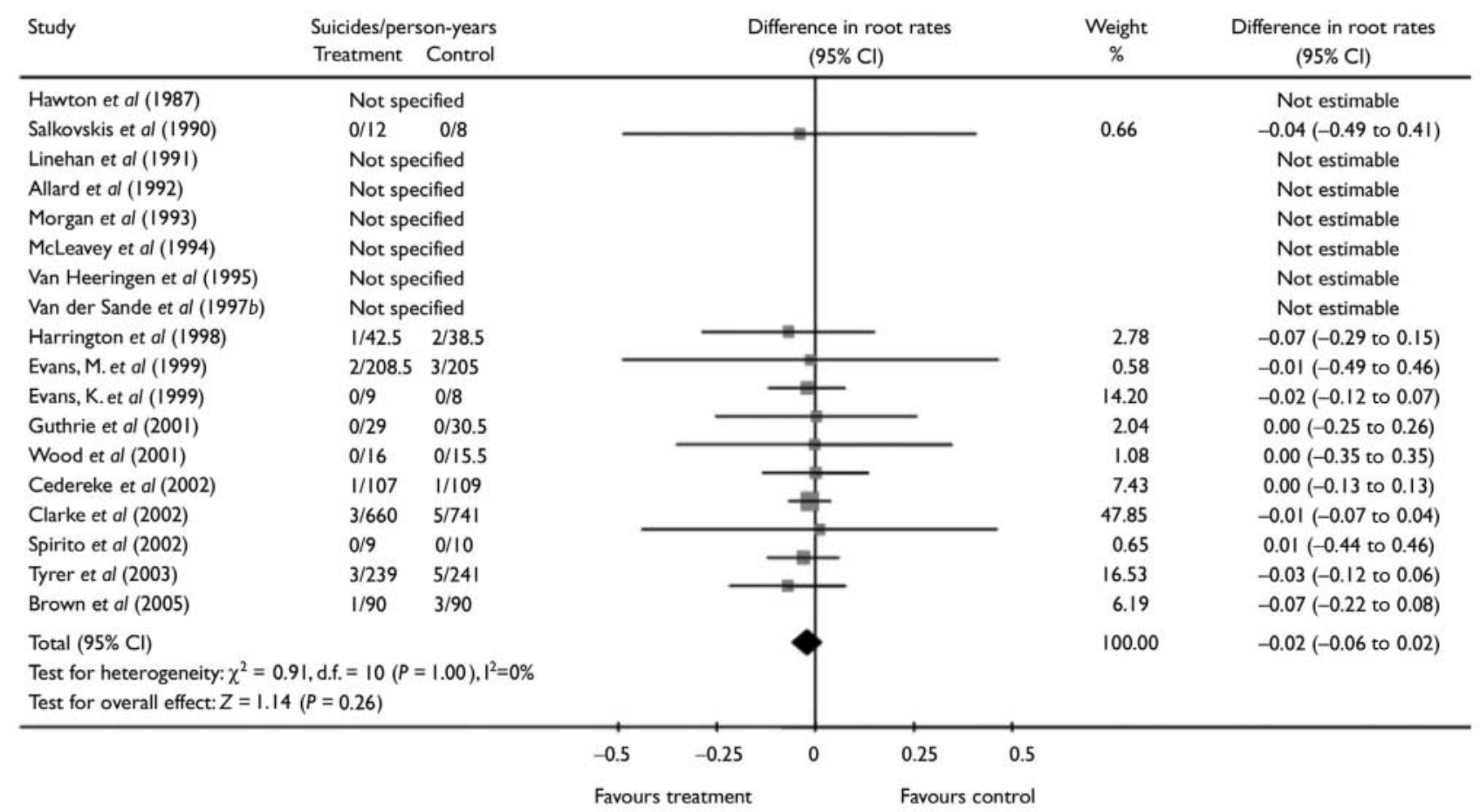

Fig. 4 Meta-analysis of psychosocial interventions on all-cause mortality. 


\section{Level of suicide}

Few papers provided information about how suicide deaths were ascertained. Given that a proportion of participants in each study were lost to follow-up it is possible that some suicide deaths were not recorded.

It has been suggested that participation in a trial might of itself affect patient outcomes (Rothwell, 2005). Participants in these studies all received a higher level of assessment as a result of participation in a trial, and most trials excluded people who were judged to be at high risk of subsequent suicide. Five trials we included followed participants for less than a year, but among those that followed people for 12 months the overall rate of suicide during this period was 0.016 . This is similar to the rate of 0.018 reported in cohorts of people followed up after an episode of self-harm (Owens et al, 2002).

\section{Interpretation of results}

The relative lack of statistical power in this meta-analysis means that caution needs to be used when interpreting the study findings. Many of the trials of psychosocial interventions we examined were too small to detect differences in the level of repetition of self-harm, and suicides occurred in only 11 of the 18 studies included in the review. During the course of this review we identified three trials of pharmacological interventions (Hirsch et al, 1982; Montgomery et al, 1983; Verkes et al, 1998). With a combined study population of 243 and one suicide by a member of the control group in the trial by Verkes and colleagues, a meta-analysis was not appropriate for these interventions.

A total of 4004 person-years (2002 in each arm) was sufficient to achieve power of $80 \%$ to detect a difference between a rate of suicide of $1.8 \%$ in the year following an episode of self-harm, and $50 \%$ reduction in the level of suicide among those receiving additional psychosocial interventions. Had trials demonstrated reductions in levels of suicide of the magnitude reported by Tyrer et al (2003) we would have had ample power to detect statistically significant differences in our primary outcome, with 4397.5 person-years in this meta-analysis. However, we had insufficient power to detect smaller - but still clinically significant - reductions in the level of suicide. Study power would have been increased had we been able to obtain suicide data from the nine remaining randomised trials that met our inclusion criteria. These nine trials included a combined population of 2914 participants.

None of the studies we identified set out to examine whether intervention would lead to a reduction in the rate of suicide, and the relatively low rate of suicide among people in the period following self-harm would make this an odd choice of outcome in a single trial. However, it has been argued that interventions to reduce repetition of suicidal behaviour following self-harm could have an impact on suicide rates (Gunnell \& Frankel, 1994; Mann et al, 2005), and publication of detailed information on suicides and other deaths occurring among participants in future trials of interventions for people who selfharm would enable a more precise estimate to be made of the impact of these interventions on suicide.

Because we were interested in exploring whether enhanced psychosocial treatment following self-harm affects the likelihood of subsequent suicide, we combined data on a variety of different types of psychosocial intervention. In doing so it is possible that the impacts of specific forms of intervention have been minimised. Differences in the rate of suicide in the trial of manual-assisted cognitive-behavioural therapy by Tyrer et al (2003) are of particular note. The single death (in the control arm) of the more recent trial of cognitivebehavioural therapy by Brown et al (2005) adds little to the statistical power required to examine whether this therapy reduces the subsequent rate of suicide. However, the relatively modest reduction in repetition of self-harm in such trials cautions against the view that this therapy is likely to be associated with marked reductions in levels of subsequent suicide.

Although we found no difference in rate of suicide among those randomised to standard and enhanced treatment, a trend towards a reduction in the overall rate of mortality was observed. This could be a chance finding, but it could reflect a real difference in mortality between those offered standard care and those offered additional psychosocial interventions. It is known that some suicide deaths are sometimes misclassified as due to other causes (Phillips \& Ruth, 1993; Neeleman et al, 1997). This finding emphasises the need to interpret the absence of difference in rate of suicide in studies with caution.

\section{Implications for suicide prevention}

This study did not examine the impact of standard care on levels of suicide following self-harm and it is possible that interventions currently provided by mental health services do much to limit the likelihood of suicide (US Department of Health and Human Services, 2001). However, we found little evidence to support the view that additional psychosocial treatment for people who self-harm leads to reductions in levels of suicide.

Individual randomised trials of psychosocial treatments have demonstrated statistically significant reductions in the likelihood of repetition of non-fatal selfharm, but such findings do not necessarily mean that these treatments would reduce the likelihood of subsequent suicide. We believe that these data support the view that a range of public health measures for preventing suicide should be pursued, including population-based strategies such as restricting access to means of suicide and encouraging responsible discussion of suicide in the media.

\section{ACKNOWLEDGEMENTS}

The project was funded by a grant from Imperial College London. The funder played no role in the design or conduct of the study or in the writing of this report. We are grateful to those authors who responded to our request for additional study data.

\section{REFERENCES}

Allard, R., Marshall, M. \& Plante, M. C. (1992)

Intensive follow-up does not decrease the risk of repeat suicide attempts. Suicide and Life Threatening Behavior, 22, 303-314.

Brown, G. K., Ten Have, T., Henriques, G. R., et al (2005) Cognitive therapy for the prevention of suicide attempts: a randomized controlled trial. JAMA, 294 563-570.

Cedereke, M., Monti, K. \& Ojehagen, A. (2002)

Telephone contact with patients in the year after a suicide attempt: does it affect treatment attendance and outcome? A randomised controlled study. European Psychiatry, 17, 82-91.

Clarke, T., Baker, P., Watts, C. J., et al (2002) Selfharm in adults: a randomised controlled trial of nurseled management versus routine care only. Journal of Mental Health, II, 167-176.

Department of Health (2002) National Suicide Prevention Strategy for England. Department of Health.

Egger, M., Davey Smith, G., Schneider, M., et al (1997) Bias in meta-analysis detected by a simple, graphical test. BMJ, 315, 629-634.

Evans, K., Tyrer, P., Catalan, J., et al (1999) Manualassisted cognitive-behaviour therapy (MACT): a randomized controlled trial of a brief intervention with bibliotherapy in the treatment of recurrent deliberate self-harm. Psychological Medicine, 29, 19-25. 
Evans, M. O., Morgan, H. G., Hayward, A., et al (1999) Crisis telephone consultation for deliberate selfharm patients: effects on repetition. British Journal of Psychiatry, 175, 23-27.

Gunnell, D. \& Frankel, S. (1994) Prevention of suicide: aspirations and evidence. BMJ, 308, 1227-1233.

\section{Guthrie, E., Kapur, N., Mackway-Jones, K., et al} (200I) Randomised controlled trial of brief psychological intervention after deliberate self poisoning. $B M \mid, 323,135-138$.

\section{Harrington, R., Kerfoot, M., Dyer, E., et al (1998)}

Randomized trial of a home-based intervention for children who have deliberatly poisoned themselves. Journal of the American Academy of Child and Adolescent Psychiatry, 37, 512-518.

Hawton, K., McKeown, S., Day, A., et al (1987) Evaluation of out-patient counselling compared with general practitioner care following overdoses. Psychological Medicine, 17, 75I-76I.

\section{Hawton, K., Townsend, E., Arensman, E., et a} (2000) Psychosocial versus pharmacological treatments for deliberate self-harm. Cochrane Database of Systematic Reviews, issue 3. Update Software.

Hirsch, S. R., Walsh, C. \& Draper, R. (1982) Parasuicide. A review of treatment interventions. Journal of Affective Disorders, 4, 299-3II.

Huffman, M. D. (1984) Miscellanea: an improved approximate two-sample (Poisson) test. Applied Statistics, 33, 224-226.

Jenkins, G. R., Hale, R., Papanastassiou, M., et al (2002) Suicide rate 22 years after parasuicide: cohort study. BMJ, 325, 1155 .

Lewis, G., Hawton, K. \& Jones, P. (1997) Strategies for preventing suicide. British Journal of Psychiatry, I7I, $351-354$

Linehan, M. M., Armstrong, H. E., Suarez, A., et a (1991) Cognitive-behavioral treatment of chronically parasuicidal borderline patients. Archives of General Psychiatry, 48, 1060-1064.

Mann, J. J., Apter, A., Bertolote, J., et al (2005) Suicide prevention strategies: a systematic review. JAMA, 294, 2064-2074.

McLeavey, B. C., Daly, R. J., Ludgate, J.W., et al (1994) Interpersonal problem-solving skills training in the treatment of self-poisoning patients. Suicide and Life Threatening Behavior, 24, 382-394.

Moher, D., Cook, D. J., Eastwood, S., et al (2000) Improving the quality of reports of meta-analyses of

MIKE J. CRAWFORD, MD, OLIVIA THOMAS, BSc, Faculty of Medicine, Imperial College London; NUSRAT KHAN, MRCPsych, Central and North West London Mental Health NHS Trust; ELENA KULINSKAYA, PhD, Faculty of Medicine, Imperial College London, London, UK

Correspondence: Dr M. J. Crawford, Department of Psychological Medicine, Claybrook Centre, 37 Claybrook Road, London W6 8LN, UK. Tel: +44 (0)207386 1233; fax: +44 (0)207386 1216; e-mail: m.crawford@imperial.ac.uk

(First received 20 April 2006, final revision 20 April 2006, accepted 2 June 2006)

randomised controlled trials: the QUOROM statement. QUOROM Group. British Journal of Surgery, 87, 1448-1454

Montgomery, S. A., Roy, D. \& Montgomery, D. B. (1983) The prevention of recurrent suicidal acts. British Journal of Clinical Pharmacology, 15, 183S-188S.

Morgan, H. G., Jones, E. M. \& Owen, J. H. (1993) Secondary prevention of non-fatal deliberate self-harm. The green card study. British Journal of Psychiatry, $\mathbf{1 6 3}$, III-II2.

Neeleman, J., Mak, V. \& Wessely, S. (1997) Suicide by age, ethnic group, coroners' verdicts and country of birth. A three-year survey in inner London. British journal of Psychiatry, 171, 463-467.

Owens, D., Horrocks, J. \& House, A. (2002) Fatal and non-fatal repetition of self-harm: systematic review. British Journal of Psychiatry, 18I, 193-199.

Phillips, D. P. \& Ruth, T. E. (1993) Adequacy of official suicide statistics for scientific research and public policy Suicide and Life Threatening Behavior, 23, 307-319.

Rothwell, P. M. (2005) External validity of randomised controlled trials: 'to whom do the results of this trial apply?'. Lancet, 365, 82-93.

Salkovskis, P. M., Atha, C. \& Storer, D. (1990) Cognitive-behavioural problem solving in the treatment of patients who repeatedly attempt suicide. A controlled trial. British Journal of Psychiatry, 157, 871-876.

Spirito, A., Boergers, J., Donaldson, D., et al (2002) An intervention trial to improve adherence to community treatment by adolescents after a suicide attempt. Journal of the American Academy of Child and Adolescent Psychiatry, 4I, 435-442.

Tyrer, P., Thompson, S., Schmidt, U., et al (2003) Randomized controlled trial of brief cognitive behaviour therapy versus treatment as usual in recurrent deliberate self-harm: the POPMACT study. Psychological Medicine, 33, 969-976.

US Department of Health and Human Services (200I) National Strategy for Suicide Prevention: Goals and Objectives for Action. DHHS.

Van der Sande, R., Buskens, E., Allart, E., et al (1997a) Psychosocial intervention following suicide attempt: a systematic review of treatment interventions. Acta Psychiatrica Scandinavica, 96, 43-50.

Van der Sande, R., van Rooijen, L., Buskens, E., et al (1997b) Intensive in-patient and community intervention versus routine care after attempted suicide. A randomised controlled intervention study. British Journal of Psychiatry, I7I, 35-4I.

Van Heeringen, C., Jannes, S., Buylaert, W., et al (1995) The management of non-compliance with referral to out-patient after-care among attempted suicide patients: a controlled intervention study. Psychological Medicine, 25, 963-970.

Verkes, R. J., Van der Mast, R. C., Hengeveld, M.W., et al (1998) Reduction by paroxetine of suicidal behavior in patients with repeated suicide attempts but not major depression. American Journal of Psychiatry, 155, 543-547.

Wood, A., Trainor, G., Rothwell, J., et al (200I) Randomized trial of group therapy for repeated deliberate self-harm in adolescents. Journal of the American Academy of Child and Adolescent Psychiatry, 40 1246-1253.

World Health Organization (2002) Suicide Prevention in Europe: The WHO European Monitoring Survey on National Suicide Prevention Programmes and Strategies. WHO Regional Office for Europe.

World Health Organization (2005) Suicide Huge but Preventable Public Health Problem. http: //www.who. int/ mediacentre/news/releases/2004/pr61/en/. 